\title{
Tychoparthenogenesis and mixed mating in natural populations of the mayfly Stenonema femoratum
}

\author{
SHELLEY L. BALL \\ Division of Biological Sciences, University of Missouri, Columbia, Missouri, 65211-7400, U.S.A.
}

\begin{abstract}
Tychoparthenogenesis is a breeding system characterized by low population mean hatching success (usually $<10 \%$ ) of unfertilized eggs from females of typically sexually reproducing species. I used progeny-array analysis to estimate outcrossing and parthenogenetic rates for two tychoparthenogenetic populations of the mayfly, Stenonema femoratum. Based on multilocus outcrossing rate estimates $\left(t_{\mathrm{m}}\right)$, populations exhibited moderate rates of tychoparthenogenetic reproduction (population LD: $1-t_{\mathrm{m}}=0.266$; population RBG: $\left.1-t_{\mathrm{m}}=0.495\right)$. Differences between multilocus and average single-locus outcrossing rates indicated some biparental inbreeding in population LD, but not in population RBG. Family outcrossing rates ranged from 0 to 1.0 , indicating mixed mating in which some females generated a mixture of sexually and parthenogenetically produced offspring. Outcrossing rates showed substantial heterogeneity among families. Correlation with paternity was high, indicating that outcrossed sibs within families were sired by the same father. Progeny-array sex ratios were significantly female biased for both populations and did not differ significantly between populations. However, family outcrossing rate was not significantly correlated with family sex ratio. Results indicate that substantial amounts of parthenogenetic reproduction are occurring in these natural $S$. femoratum populations and that some females produce mixed broods of sexually and parthenogenetically produced offspring.
\end{abstract}

Keywords: isozyme, mayfly, outcrossing, progeny array, Stenonema femoratum, tychoparthenogenesis.

\section{Introduction}

Tychoparthenogenesis is characterized by low hatching success of unfertilized eggs from females of typically sexually reproducing species (White, 1973; Bell, 1982; Suomalainen et al., 1987). Population mean hatching success of unfertilized eggs is generally less than 10 percent, although variation among females within populations can be extremely large (Huff \& McCafferty, 1974; Templeton, 1982; Ball, 2000). Because hatching success is low, tychoparthenogenesis has often been viewed as 'accidental' or 'occasional' parthenogenesis and has been dismissed as artificial parthenogenesis unimportant to fitness (Maynard Smith, 1978; Mingo, 1978; Brittain, 1982). However, if tychoparthenogenetic reproduction occurs in natural populations, the frequency of tychoparthenogenetic reproduction, a

Correspondence and present address: Department of Biology, Bates College, 44 Campus Ave., Lewiston, Maine, U.S.A.

E-mail: sball@bates.edu female's tychoparthenogenetic capacity (i.e. proportion of her unfertilized eggs hatching), and the proportion of females in a population producing offspring through tychoparthenogenesis can all potentially have significant effects on individual fitness as well as on the genetic structure and evolutionary fate of populations. Although tychoparthenogenesis has been documented in a diversity of invertebrates (Bell, 1982), it has been largely ignored by evolutionary biologists. Detailed genetic studies of several Drosophila species provide the bulk of our understanding about tychoparthenogenesis (summarized in Templeton, 1982); however, these studies have been limited to the laboratory and provide no information on the extent to which tychoparthenogenetic reproduction occurs in nature.

Mixed-mating models developed for self-fertilizing plants have been used to estimate outcrossing and selfing rates in several self-fertilizing hermaphroditic animals (Jarne, 1995; Städler et al., 1995; Cohen, 1996; Viard et al., 1996, 1997). These models can also be appropriate for estimating outcrossing and 
parthenogenetic rates for tychoparthenogens. Whereas most obligate parthenogens are apomictic (mitotic parthenogens), all known tychoparthenogens are automictic (meiotic parthenogens; Templeton, 1982). There are several different types of automixis, which differ in the stage of meiosis during which diploidy is restored. Like self-fertilization, automixis results in a loss of heterozygous genotypes with each generation (Bell, 1982; Templeton, 1982). Therefore, mixed-mating models developed for self-fertilizing plants can be used to estimate the amount of tychoparthenogenetic reproduction occurring in natural populations.

Parthenogenesis has been documented in 24 North American and 35 European mayfly species (DeGrange, 1960; Huff \& McCafferty, 1974; Mingo, 1978; Sweeney \& Vannote, 1987; Harker, 1997). Tychoparthenogenesis has been documented in the heptageniid mayfly, Stenonema femoratum. Ball (2000) showed that the tychoparthenogenetic capacity of females varied among habitats, with population mean hatching success of unfertilized eggs in temporary streams (mean $\pm \mathrm{SE}=$ $7.6 \% \pm 1.5 \%$ ) nearly double that of permanent streams $(3.9 \% \pm 0.8 \%)$ or lakes $(3.8 \% \pm 0.8 \%)$.

The purpose of this study was to determine whether tychoparthenogenetic reproduction occurs in natural populations of the mayfly, Stenonema femoratum, or whether it is simply a case of artificial parthenogenesis induced in the laboratory. I used progeny-array analysis to estimate the relative proportions of tychoparthenogenesis and outcrossing from two natural populations. If substantial amounts of parthenogenetic reproduction occur in these populations, then tychoparthenogenesis may have important influences on population genetic structure and evolution in these populations. I also estimated family outcrossing rates to determine whether individual females produced mixed broods of sexually and parthenogenetically produced progeny. Finally, I tested whether progeny-array sex ratio was correlated with the rate of tychoparthenogenesis because tychoparthenogenetically reproducing females should produce female-biased progeny arrays.

\section{Methods}

\section{Female collection and nymph rearing}

Adult females for outcrossing rate analysis were collected from two populations in central Missouri, USA: Little Dixie (LD), a lake population (Callaway County: $\left.38^{\circ} 55^{\prime} \mathrm{N}, 9^{\circ} 06^{\prime} \mathrm{W}\right)$ and Rock Bridge Gans Creek (RBG), a temporary stream population (Boone County: $\left.38^{\circ} 52^{\prime} \mathrm{N}, 92^{\circ} 20^{\prime} \mathrm{W}\right)$. Ovipositing females were captured with an insect net as they flew a few $\mathrm{cm}$ above the water surface repeatedly dipping the tip of their abdomens into the water to release eggs - a behaviour that is distinctly different from male 'skimming' behaviour during swarming. I collected females only after I had seen them dip their abdomen at least once. This ensured that females were ovipositing and not simply patrolling the area looking for mating swarms. Most ovipositing females were collected within $15 \mathrm{~min}$ of the appearance of the first ovipositing female. This was done in an effort to collect females immediately after they began oviposition and to minimize collecting females that may have already laid a large proportion of their eggs. Oviposition occurred around 6 p.m., during daylight, and therefore ovipositing females could be identified easily. In the RBG population females generally began oviposition $45 \mathrm{~min}$ prior to dusk and were more difficult to see. Therefore, a 500, 000 candle power halogen hand-held spotlight was used to illuminate the water surface to identify ovipositing females. On each night I captured as many ovipositing females as possible. A total of 66 females was caught from the LD population, from April 6th to May 2nd, 1999. Of these, 57 females laid eggs that hatched and produced at least a single offspring. At the RBG population, 126 ovipositing females were caught, from the 2nd to the 20th of May. Of these, 63 females laid eggs that hatched and produced at least a single offspring. Fewer RBG females laid eggs, presumably because some females had laid most of their eggs before being captured.

Captured females were transferred to small plastic vials containing $15 \mathrm{~mL}$ carbon-filtered tap water, taken to the laboratory, and placed in random order on shelves, approximately $25 \mathrm{~cm}$ below $\mathrm{Gro}^{\mathrm{TM}}$ lights. Lights were kept on a 16L:8D photoperiod. Most females released their eggs into the water-filled vials immediately, but some females released eggs over a period of $2-3$ days in the laboratory. Females remained in vials until they died and, if they had laid eggs, were stored at $-80^{\circ} \mathrm{C}$ for later genetic analysis. Eggs hatched $\approx 7$ days after being laid. Vials were checked daily and hatchlings were added to their family-rearing containers. I monitored each vial for three weeks because parthenogenetically produced eggs take longer $(\approx 12$ days) to hatch (Ball, 2000). Family-rearing containers contained a 1-cm deep layer of natural aquarium gravel and $2 \mathrm{~L}$ of carbon-filtered tap water. Two to three ceramic tiles with miniature clay flower pots were added to each container to provide nymphs with refuge and a food source. Tiles each had a small $(4 \mathrm{~cm}$ diameter) clay flower pot, containing a $0.1-\mathrm{M}$ potassium phosphate solution in solidified agar, inverted and glued to the upper surface of the tile. Potassium phosphate provided nutrients for algae and diatoms growing on the flower pot surface; both of these are eaten by $S$. femoratum nymphs. Prior to being added to mayfly-rearing con- 
tainers, pots and tiles were aged for 2-4 weeks in small plastic wading pools containing water, dry leaf litter, and algae from both the LD and RBG populations. Wading pools were kept outside and screening was placed over each pond to exclude predatory aquatic insects and to prevent mayflies from laying eggs in ponds.

Five grams of pond-aged deciduous leaf litter was also added to each family-rearing container to provide more nutrients for algal and diatom growth. Water was changed weekly except for the first two weeks, in which hatchlings were too small to be seen. This prevented the inadvertent transfer of hatchlings, which might stick to my hands or glassware, between containers. Every 3-4 weeks, pond-aged deciduous leaf litter was added to rearing containers to supply nutrients for algae and diatoms. Tiles and pots were replaced once over the course of the experiment. This was done only when nymphs were large enough to be seen and therefore would not be removed inadvertently. Once nymphs had reached a total body length (head to tip of the abdomen) of at least $1 \mathrm{~cm}$, they were collected and placed in individually labelled plastic microcentrifuge tubes and frozen at $-80^{\circ} \mathrm{C}$. Rearing containers were checked weekly and appropriately sized nymphs collected. Laboratory rearing of nymphs continued until October 1st, when the last nymphs reached the appropriate size. Prior to electrophoresis, nymphs were thawed and examined under a dissecting microscope to determine their gender. Males are distinguishable by the developing genital forceps on the tip of the abdomen. These structures are discernible even on very young nymphs; however, in a few cases sex could not be confidently ascertained.

\section{Electrophoresis}

Isozymes were separated using cellulose acetate gel electrophoresis. I scored four loci: arginine kinase (Ark: E.C. 2.7.3.3), glucose-6-phosphate isomerase (Pgi: E.C. 5.3.1.9), peptidase (leucine-alanine) (Pep: E.C. 3.4.11) and phosphoglucomutase (Pgm: E.C. 5.4.2.2). Loci were resolved in one of two continuous electrode buffers, Tris-glycine, $\mathrm{pH} 8.5$ (Pgm, Pgi, Pep) and Tris-citrate, pH 7.0 (Ark). Electrode buffer and stain recipes were taken from Richardson et al. (1986) and Hebert \& Beaton (1993). Tris-glycine gels were run at $200 \mathrm{~V}$ (2 mA) for 15-20 min, except for Pep which was run for $30 \mathrm{~min}$; Tris-citrate gels were run at $140 \mathrm{~V}(5-10 \mathrm{~mA})$ for $25 \mathrm{~min}$. Whole nymphs and adult females were homogenized in 8-40 $\mu \mathrm{L}$ (depending on size) of ice-cold grinding buffer consisting of a $1: 1$ solution of $0.2 \mathrm{M}$ Tris$\mathrm{HCl}(\mathrm{pH} 8.0)$ and $10 \mathrm{mg} / \mathrm{mL}$ NADP. Samples were centrifuged on a tabletop microcentrifuge for $20 \mathrm{~s}$.
Approximately $2 \mu \mathrm{L}$ of each sample was loaded into a sample well plate (Helena Laboratories), containing samples from the mother and 10-11 of her randomly selected offspring. Line-up gels were used extensively to confirm that alleles were scored consistently across populations. Multilocus genotypes were obtained for $17 \mathrm{LD}$ and $15 \mathrm{RBG}$ families.

\section{Data analysis}

Population-level multilocus $\left(t_{\mathrm{m}}\right)$ and average singlelocus $\left(t_{\mathrm{s}}\right)$ outcrossing rates were estimated using the mixed-mating model of Ritland \& Jain (1981), Ritland (1986), and Ritland (1990). I used the MLTR computer program (version 1.1, K. Ritland 1996) to obtain maximum likelihood estimates of outcrossing rates and associated parameters. I used the expectation-minimization (EM) algorithm to estimate parameters because of its greater stability (Ritland, 1983; 1986). The maximum number of iterations was allowed and variances were estimated for each parameter using 1000 bootstraps. For population-level estimates, the family was used as the unit of resampling. The parental inbreeding coefficient, $F$, was also estimated. Male and female allele frequencies were jointly estimated with $t$. Family outcrossing rates were also estimated using individual progeny as the unit of resampling. Male gene frequencies were jointly estimated with family outcrossing rates to avoid biases introduced when $p$ varies among families (Ritland \& Ganders, 1987). Variances were estimated by bootstrapping 200 times. I also examined the influence of each locus on estimates of $t_{\mathrm{m}}$, by re-running the MLTR model, as described above, but including only three of the four loci. A different locus was excluded each time.

MLTR was also used to estimate $r_{\mathrm{s}}$ and $r_{\mathrm{p}}$, the correlation of selfing and the correlation of paternity, respectively. Correlation of selfing (in this case, correlation of parthenogenesis) describes the among-family variation in mating system, with $r_{\mathrm{s}}=0$ indicating homogeneity of outcrossing rates among maternal parents, and $r_{\mathrm{s}}=1$ indicating heterogeneity among outcrossing rates (i.e. siblings are entirely derived from either selfing or outcrossing). I used the two-sample Kolmogorov-Smirnoff test to compare the distribution of family outcrossing rates for the two populations. The correlation of outcrossed paternity is an estimate of the proportion of full-sibs among outcrossed sibs within a family and indicates whether offspring are the result of single or multiple paternity.

For each family I recorded the sex of each individual and calculated sex ratio ( $\%$ female). I calculated population mean sex ratio by taking the mean percentage female of all families in each population. I used Chi-squared goodness of fit to test if sex ratios deviated 
significantly from 1:1 F:M and used Pearson correlation to determine if sex ratio was related to outcrossing rate separately for each population.

\section{Results}

Populations showed moderate rates of parthenogenetic reproduction $\left(1-t_{\mathrm{m}}\right)$, with the RBG population showing a higher rate compared to LD (Table 1). Populations also differed in the proportion of parthenogenetic families. Only $6 \%$ of families from LD contained parthenogenetically produced progeny, whereas $23 \%$ of families from RBG contained such progeny. Values of parental $F$ were low, indicating that the parents themselves were not highly inbred (Table 1). Comparisons of multilocus and single-locus estimates of $t_{\mathrm{m}}$ indicated no biparental inbreeding in RBG, but some biparental inbreeding in LD (Table 1). Estimation of male and female parent allele frequencies for each locus are given in Table 2. In both populations, the Pgm locus was dominated by a single allele that occurred at high frequency (LD: $P g m$, allele 2=0.837; RBG: $P g m$, allele $2=0.913$ ), whereas all other loci exhibited moderate to high levels of polymorphism.

Sensitivity analysis, in which $t_{\mathrm{m}}$ was calculated using three of the four loci, showed that estimates were comparable for all loci except $P g i$ in the LD population (Table 3). Exclusion of $P g i$ from estimation of $t_{\mathrm{m}}$ resulted in a substantially lower value of $t_{\mathrm{m}}$, indicating that $P g i$ was biasing the population outcrossing rate upward. Similarly, exclusion of $P g i$ from $t_{\mathrm{m}}$ estimates in RBG also showed a substantial influence of $P g i$ on $t_{\mathrm{m}}$ estimated across all four loci. However, in contrast to the LD population, exclusion of $P g i$ resulted in a substantial increase in $t_{\mathrm{m}}$, relative to the other three locus estimates (Table 3 ).

Correlation of parthenogenesis $\left(r_{\mathrm{s}}\right)$ was large for both populations, indicating significant heterogeneity in outcrossing rates among families (Table 1). RBG exhibited a slightly bimodal distribution of outcrossing rates, showing that many families were either primarily outcrossed or primarily the result of parthenogenesis (Fig. 1). However, the distribution of family outcrossing rates did not differ significantly between the two populations (Kolmogorov-Smirnoff two-sample test,
Table 2 Male and female parent allele frequencies for populations LD and RBG, estimated using MLTR. Standard deviations are given in parentheses

\begin{tabular}{|c|c|c|c|}
\hline Locus & Allele & Male & Female \\
\hline \multicolumn{4}{|l|}{ LD } \\
\hline \multirow[t]{3}{*}{$P g m$} & 1 & $0.055(0.049)$ & $0.088(0.047)$ \\
\hline & 2 & $0.837(0.084)$ & $0.853(0.055)$ \\
\hline & 3 & $0.108(0.071)$ & $0.059(0.035)$ \\
\hline \multirow[t]{4}{*}{$P g i$} & 1 & $0.043(0.039)$ & $0.059(0.034)$ \\
\hline & 2 & $0.216(0.122)$ & $0.382(0.052)$ \\
\hline & 3 & $0.598(0.145)$ & $0.500(0.069)$ \\
\hline & 4 & $0.142(0.096)$ & $0.059(0.035)$ \\
\hline \multirow[t]{3}{*}{ Pep } & 1 & $0.184(0.089)$ & $0.324(0.075)$ \\
\hline & 2 & $0.696(0.094)$ & $0.588(0.078)$ \\
\hline & 3 & $0.119(0.062)$ & $0.088(0.061)$ \\
\hline \multirow[t]{3}{*}{ Ark } & 1 & $0.173(0.105)$ & $0.265(0.087)$ \\
\hline & 2 & $0.762(0.108)$ & $0.676(0.085)$ \\
\hline & 3 & $0.065(0.052)$ & $0.059(0.042)$ \\
\hline \multicolumn{4}{|l|}{$\mathrm{RBG}$} \\
\hline \multirow[t]{3}{*}{ Pgm } & 1 & $0.073(0.070)$ & $0.100(0.063)$ \\
\hline & 2 & $0.913(0.070)$ & $0.833(0.068)$ \\
\hline & 3 & $0.015(0.004)$ & $0.067(0.038)$ \\
\hline \multirow[t]{4}{*}{$P g i$} & 1 & $0.019(0.005)$ & $0.032(0.001)$ \\
\hline & 2 & $0.243(0.153)$ & $0.323(0.075)$ \\
\hline & 3 & $0.719(0.158)$ & $0.516(0.081)$ \\
\hline & 4 & $0.019(0.005)$ & $0.129(0.071)$ \\
\hline \multirow[t]{4}{*}{ Pep } & 1 & $0.160(0.065)$ & $0.367(0.106)$ \\
\hline & 2 & $0.401(0.139)$ & $0.433(0.086)$ \\
\hline & 3 & $0.422(0.151)$ & $0.167(0.058)$ \\
\hline & 4 & $0.017(0.004)$ & $0.033(0.023)$ \\
\hline \multirow[t]{3}{*}{ Ark } & 1 & $0.016(0.004)$ & $0.100(0.055)$ \\
\hline & 2 & $0.757(0.134)$ & $0.733(0.065)$ \\
\hline & 3 & $0.227(0.133)$ & $0.167(0.064)$ \\
\hline
\end{tabular}

Table 3 Influence of each locus on population estimates of $t_{\mathrm{m}}$ determined by excluding one locus from each run of the MLTR model. Values are $t_{\mathrm{m}}$ for each population; standard deviations are given in parentheses

\begin{tabular}{lcc}
\hline Locus removed & LD & RBG \\
\hline Pgm & $0.662(0.108)$ & $0.418(0.110)$ \\
Pgi & $0.431(0.128)$ & $0.732(0.135)$ \\
Pep & $0.818(0.118)$ & $0.530(0.199)$ \\
Ark & $0.783(0.099)$ & $0.533(0.154)$ \\
\hline
\end{tabular}

Table 1 Estimates of population-level outcrossing parameters based on mixed-mating model (MLTR): parental inbreeding coefficient $(F)$, multilocus outcrossing rate $\left(t_{\mathrm{m}}\right)$, single-locus outcrossing rate $\left(t_{\mathrm{s}}\right)$, correlation of parthenogenesis $\left(r_{\mathrm{s}}\right)$, and correlation of paternity $\left(r_{\mathrm{p}}\right)$. Standard deviations are in parentheses

\begin{tabular}{lcccccc}
\hline Population & $F$ & $t_{\mathrm{m}}$ & $t_{\mathrm{s}}$ & $t_{\mathrm{m}}-t_{\mathrm{s}}$ & $r_{\mathrm{s}}$ & $r_{\mathrm{p}}$ \\
\hline LD & $0.015(0.029)$ & $0.734(0.091)$ & $0.596(0.084)$ & $0.138(0.052)$ & $0.610(0.110)$ & $0.990(0.003)$ \\
RBG & $0.061(0.058)$ & $0.505(0.119)$ & $0.523(0.040)$ & $-0.018(0.040)$ & $0.707(0.139)$ & $0.990(0.003)$ \\
\hline
\end{tabular}



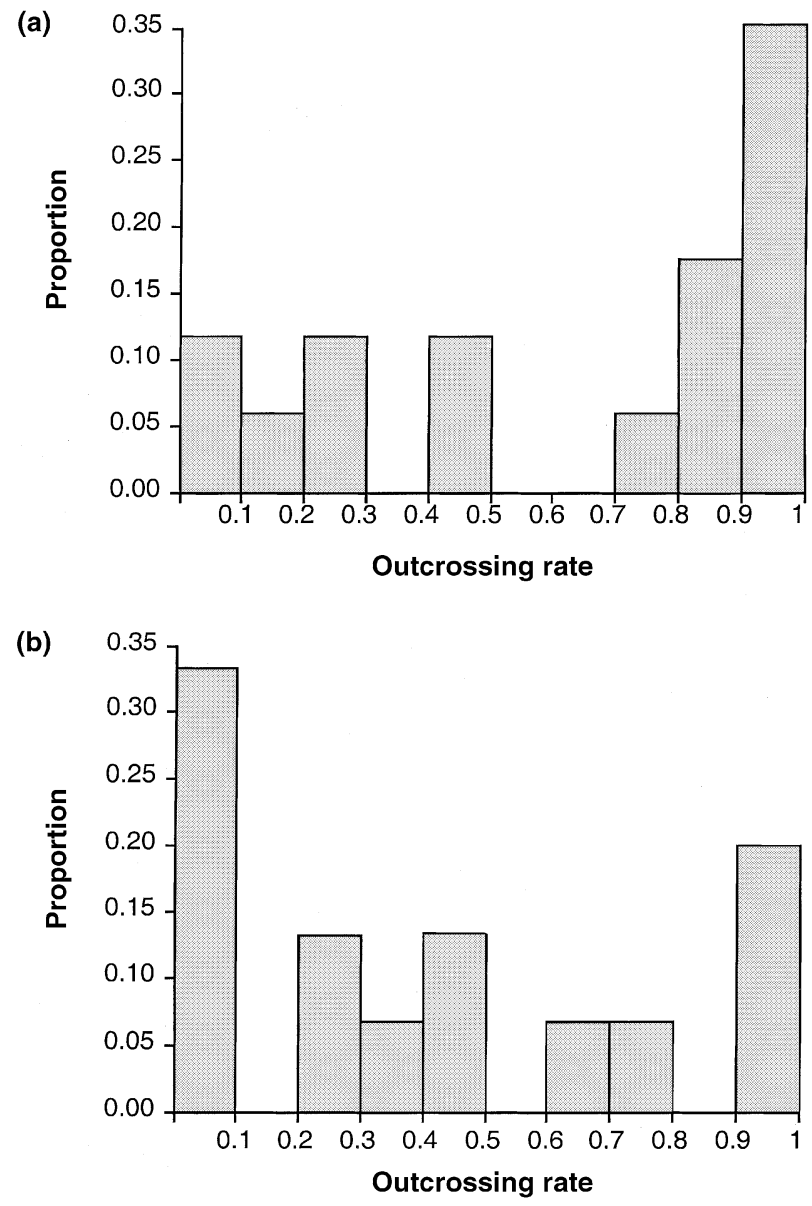

Fig. 1 Distribution of family outcrossing rates estimated using MLTR, for (a) LD and (b) RBG populations.

Table 4 Population mean sex ratio and standard deviation (SD) for Little Dixie (LD) and Rock Bridge Gans Creek (RBG), estimated from progeny arrays. Asterisks indicate significant deviations from a 1:1 male:female sex ratio

\begin{tabular}{lccc}
\hline Population & Mean \% female & SD & \multicolumn{1}{c}{ Range } \\
\hline LD & $68.9 \% *$ & 17.7 & $36.4-100.0$ \\
RBG & $72.1 \% * *$ & 10.9 & $45.5-90.0$ \\
\hline
\end{tabular}

$* \chi^{2}=25.41$, d.f. $=1, P<0.001 ; * * \chi^{2}=32.60$, d.f. $=1$, $P<0.0001$.

$D=0.329, P=0.35)$. Correlation of paternity was large for both populations, indicating that over $99 \%$ of outcrossed sibs within progeny arrays were full sibs.

If tychoparthenogenetic reproduction is occurring in these populations, as indicated by outcrossing rate analysis, then offspring from these females should exhibit female-biased sex ratios. Mean family sex ratios were significantly female biased for both populations (Table 4), however, sex ratios did not differ significantly between the two populations $(t=0.623$, d.f. $=31$, $P=0.54)$. In addition, family outcrossing rate was not significantly correlated with sex ratio for either the LD $(r=0.02, \quad$ d.f. $=14, \quad P=0.63) \quad$ or $\quad \mathrm{RBG} \quad(r=0.09$, d.f. $=14, P=0.27$ ) populations.

\section{Discussion}

Progeny array analysis clearly showed that parthenogenetic reproduction was occurring in these mayfly populations and that this was not simply a case of artificial parthenogenesis induced in the laboratory. Furthermore, the amount of tychoparthenogenetic reproduction was far greater than expected, based on previous laboratory studies showing population mean hatching success of unfertilized eggs of less than 10 percent (Ball, 2000). If, on average, females from these populations show such low parthenogenetic capabilities, how then can these populations produce approximately $30-50 \%$ of their offspring through parthenogenesis, as shown here? Based on reproductive output, sexual females should have a several-fold advantage compared to tychoparthenogens and tychoparthenogenesis should be eliminated from populations. However, females may occasionally find themselves under ecological conditions where mates are difficult or impossible to find and thus are forced to rely on their tychoparthenogenetic capabilities by laying unfertilized eggs. Under such conditions females with high parthenogenetic capacities (large proportions of unfertilized eggs hatching) will contribute more offspring to the population than sexual females or females with low parthenogenetic capacities. This greater reproductive contribution by females with high parthenogenetic capacities can explain the discrepancy between estimates of parthenogenesis based on outcrossing rate determination and hatching success of unfertilized eggs tested in the laboratory.

Although tychoparthenogenesis is defined by population mean hatching success of unfertilized eggs of typically $<10 \%$, enormous intrapopulation variation in hatching success among females has been shown. Huff \& McCafferty (1974) documented population mean hatching success of unfertilized eggs of $8 \%$ in a tychoparthenogenetic population of $S$. femoratum in Indiana. However, hatching success of unfertilized eggs within that population varied between 0.2 and $40 \%$. Extremely large variation has also been documented in several other mayfly species (see summary in Sweeney \& Vannote, 1987) and in several Drosophila species (Templeton, 1982). Ball (2000) showed extremely large variation in $S$. femoratum parthenogenetic capacity among females in the RBG population, with up to $78 \%$ of unfertilized eggs hatching for some females. If females are forced to reproduce parthenogenetically 
due to extremely low male densities, then these females with higher parthenogenetic capacities will contribute more offspring to the population. Furthermore, environmental stochasticity is likely to eliminate the few offspring produced by females with low parthenogenetic capacities. Together, these factors can explain the large discrepancy between parthenogenetic rates of $\approx 30-50 \%$, as measured by progeny array analysis, and the low $(<10 \%)$ population mean hatching success of unfertilized eggs estimated in the laboratory.

Estimates of parthenogenesis may also be influenced by offspring viability. If viability of parthenogenetically produced offspring, from females with high parthenogenetic capacities, is high compared to that of females with low parthenogenetic capacities, then females with high capacities will contribute more offspring to the population. In self-fertile plants, primarily self-fertilizing lineages often experience less inbreeding depression (i.e. higher germination success and seedling survivorship) compared to primarily outcrossing lineages (Schemske \& Lande, 1985; Dudash et al., 1997). If parthenogenetically produced offspring from mothers with low parthenogenetic capacities experience extremely low survivorship due to inbreeding depression, then only females with high parthenogenetic capacities will contribute parthenogenetic offspring to the population.

Estimates of both adult and nymph density strongly suggest that female $S$. femoratum occasionally do find themselves under conditions where mates are extremely difficult to find. Nymph densities in temporary stream populations, such as the RBG population, were extremely low compared to permanent stream and lake populations (Ball, 2000). Moreover, temporary streams dry by mid summer in Missouri, forming small isolated pools. This habitat fragmentation coupled with the extremely short adult lifespan (24-48 h) of these mayflies may make it difficult for females to find mates. Habitat fragmentation through stream drying may also result in stochastic differences in sex ratio among pools and lead to rarity or absence of males in a given pool (Templeton, 1982). Adult mayfly densities measured over the emergence season showed that early and late in the season densities were often extremely low. In March and October, light trap samples of adults often contained fewer than 10 individuals (Ball, 2000). In addition, male mating swarms observed during the day, in March and October, were extremely small, consisting only of several males. Typically, S. femoratum females begin oviposition while male mating swarms are present. However, in early March and late October I often observed small numbers of females ovipositing, yet no male mating swarms were observed in the area. This lack of available mates early and late in the season and the brief adult lifespan may force females to reproduce parthenogenetically.

Temporal variation in parthenogenetic capacities of females within populations may also contribute to the discrepancy between these estimates of parthenogenesis. Ball (2000) showed that tychoparthenogenetic capacity varied among sampling dates in some populations. In 1997, population mean hatching success of unfertilized eggs from females in the RBG population ranged from approximately $2.5 \%$ in mid July to nearly $16 \%$ in late June. Therefore, outcrossing and parthenogenetic rates reported here may have been measured at a time when females with high parthenogenetic capacities were more prevalent in these populations.

The discrepancy between estimates of parthenogenesis may also be influenced by the type of automixis. Gamete duplication results in homozygosity at all loci in one generation while central fusion, like self-fertilization, can result in a $50 \%$ loss of heterozygosity with each generation (White, 1973; Templeton, 1982; Soumalainen et al., 1987). Stalker (1954) showed that some tychoparthenogenetic Drosophila populations consisted of a mixture of females using different types of automixis, but that populations usually converged upon a single automictic mechanism. Variation in the type of automixis among females in these S. femoratum populations may explain the discrepancy between estimates of parthenogenesis. The MLTR model estimates outcrossing rates based on genotypic frequencies expected under self-fertilization. Therefore, tychoparthenogenetic rates would be overestimated for females that produced offspring through gamete duplication. Preliminary studies with a small number of progeny arrays from virgin $S$. femoratum females, from both the LD and RBG populations, indicated that some heterozygous females produced both homozygous and heterozygous offspring, which is not consistent with gamete duplication or other types of automixis that enforce complete homozygosity. However, cytogenetic studies are needed to determine whether females within populations vary in the type of automixis that occurs.

Both populations showed significant heterogeneity in outcrossing rates among families. This was particularly pronounced for RBG, as shown by the somewhat bimodal distribution of outcrossing rates. This pattern may result if females experience different environmental conditions, which influence reproductive mode. Variation in outcrossing rate has been shown to vary with water temperature, in the snail Bulinus truncatus (Woolhouse \& Chandiwana, 1989; Schrag \& Read, 1992; Schrag et al., 1994). Female mayflies from which progeny arrays were generated, were caught over a period of approximately one month. If tychoparthenogenesis is influenced to a large degree by current 
environmental conditions, then varying environmental conditions over the month may have contributed to variation in outcrossing rate.

If some offspring in these populations are produced parthenogenetically, then families should be female biased, with the magnitude of the bias proportional to the amount of tychoparthenogenetic reproduction. Mean family sex ratio was significantly female biased for both populations, further supporting the finding that tychoparthenogenetic reproduction was occurring in these populations. However, the lack of significant correlation between sex ratio and outcrossing rate in either population could result from greater female nymph mortality during rearing, thereby reducing the magnitude of any female biased primary sex ratios. Primary sex ratios would need to be determined to better understand why sex ratio was not significantly correlated with outcrossing rates.

The finding that substantial amounts of tychoparthenogenetic reproduction occurred in these natural $S$. femoratum populations is significant because the maintenance of tychoparthenogenesis seems difficult to explain, because of the low population mean hatching success of unfertilized eggs. Although females probably reproduce sexually when mates are abundant, the ability to produce offspring parthenogenetically may be advantageous early and late in the season, when mates are difficult to find. This occasional advantage may be great enough to prevent selection from eliminating parthenogenesis from populations. Furthermore, mixed broods may provide a type of bet-hedging strategy for females by producing genetically variable offspring, which may be advantageous in changing environments, whereas parthenogenetically produced offspring may inherit locally adapted gene complexes which are fine-tuned for the current environment. Further work is needed to compare the relative fitness of sexually and parthenogenetically produced offspring under different ecological conditions and to further understand why tychoparthenogenesis persists in populations despite such low population-level rates of offspring production.

\section{Acknowledgements}

I thank Gayle and Patrick Birchfield for their help in collecting ovipositing females and to Tim Holtsford and Paul Hebert for their help with electrophoresis. Thanks also to G. Birchfield, C. Galen, T. Holtsford, S. Muse, M. Parris, B. Poulton, B. Sites and two anonymous reviewers for constructive comments that greatly improved this manuscript. I am extremely grateful to the following funding sources for their support: Sigma Xi Grant-in-aid of Research, American Museum of Natural History (Theodore Roosevelt
Award), Sigma Delta Epsilon/Graduate Women in Science (Eloise Gerry Award), University of Missouri Columbia Alumni Association Faculty Incentive Grant, and NSF Doctoral Dissertation Improvement Grant (DEB 9801568).

\section{References}

BALL, S. L. 2000. Evolutionary Ecology and Population Genetics of Tychoparthenogenesis in the Mayfly, Stenonema femoratum (Ephemeroptera: Heptageniidae). PhD Thesis, University of Missouri - Columbia.

BELL, G. 1982. The Masterpiece of Nature: The Evolution and Genetics of Sexuality. University of California Press, Berkeley, California.

BritTAin, J. E. 1982. Biology of mayflies. Ann. Rev. Entomology, 27, 119-147.

COHEN, C. S. 1996. The effects of contrasting modes of fertilization on levels of inbreeding in the marine invertebrate genus Corella. Evolution, 50, 1896-1907.

DEGRANGE, C. 1960. Recherches sur la reproduction des Ephemeropteres. Travaille du laboratoire d'hydrobiologie et de pisciculture de l'Université Grenoble, 50/51, 7-193.

DUDASH, M. R., CARR, D. E. AND FENSTER, C. B. 1997. Five generations of enforced selfing and outcrossing in Mimulus guttatus: inbreeding depression variation at the population and family level. Evolution, 51, 54-65.

HARKER, J. E. 1997. The roles of parthenogenesis in the biology of two species of mayfly (Ephemeroptera). Freshwater Biol., 37, 287-297.

HEBERT, P. D. N. AND BEATON, M. J. 1993. Methodologies for Allozyme Analysis Using Cellulose Acetate Electrophoresis: a Practical Handbook. Helena Laboratories, Beaumont, TX, USA.

HUFF, B. L. AND MCCAFFERTy, w. P. 1974. Parthenogenesis and experimental reproductive biology in four species of the mayfly genus Stenonema. The Wasmann J. Biol., 32, 247-254.

JARNE, P. 1995. Mating system, bottlenecks, and genetic polymorphism in hermaphroditic animals. Genet. Res., 65 , 93-207.

MAYNARD SMITH, J. 1978. The Evolution of Sex. Oxford University Press, Oxford.

MINGO, T. M. 1978. Parthenogenesis in the mayfly Stenacron interpunctatum frontale (Burks) (Ephemeroptera: Heptageniidae). Entomol. News, 89, 46-50.

RICHARDSON, B. J., BAVERSTOCK, P. R. AND ADAMS, M. 1986. Allozyme Electrophoresis: A Handbook for Animal Systematics and Population Studies. Academic Press, San Diego, CA.

RITLAND, K. 1983. Estimation of mating systems. In: Tanksley, S. D. and Orton, T. J. (eds) Isozymes in Plant Genetics and Breeding, part A. Elsevier Science Publishers, Amsterdam.

RITLAND, K. 1986. Joint maximum likelihood estimation of genetic and mating structure using open-pollinated progenies. Biometrics, 42, 25-43.

RITLAND, K. 1990. A series of FORTRAN computer programs for estimating plant mating systems. J. Hered., 81, 235-237. 
RITLAND, K. AND GANDERS, F. R. 1987. Covariation of selfing rates with parental gene fixation indices within populations of Mimulus guttatus. Evolution, 41, 760-771.

RITLAND, K. AND JAIN, S. 1981. A model for the estimation of outcrossing rate and gene frequencies using $\mathrm{n}$ independent loci. Heredity, 47, 35-52.

SCHEMSKE, D. W. AND LANDE, R. 1985. The evolution of selffertilization and inbreeding depression in plants. II. Empirical observations. Evolution, 39, 41-52.

SCHRAG, S. J. AND READ, A. F. 1992. Temperature determination of male outcrossing ability in a simultaneous hermaphrodite. Evolution, 46, 1698-1707.

SCHRAG, S. J., MOOERS, A. O., NDIFON, G. T. AND READ, A. F. 1994. Ecological correlates of male outcrossing ability in a simultaneous hermaphrodite snail. Am. Nat., 143, 636-655.

STÄDLER, T., WEISNER, S. AND STREIT, B. 1995. Outcrossing rates and correlated matings in a predominantly selfing freshwater snail. Proc. R. Soc. B, 262, 119-125.

STAlker, H. D. 1954. Parthenogenesis in Drosophila Genetics, 39, 4-34.

SUOMALAINEN, E., SAURA, A. AND LOKKI, J. 1987. Cytology and Evolution in Parthenogenesis. CRC Press, Boca Raton, FL.
SWEENEY, B. W. AND VANNOTE, R. L. 1987. Geographic parthenogenesis in the stream mayfly Eurylophella funeralis in eastern North America. Holarctic Ecol., 10, 52-59.

TEMPLETON, A. R. 1982. The prophesies of parthenogenesis. In: Dingle, H. and Hegmann, J. P. (eds) Evolution and Genetics of Life Histories. Springer-Verlag, New York.

VIARD, F., BREMOND, P. R., LABBO, R., JUSTY, F. ET AL. 1996. Microsatellites and the genetics of highly selfing populations in the freshwater snail Bulinus truncatus. Genetics, 142, 1237-1247.

VIARD, F., DOUMS, C. AND JARNE, P. 1997. Selfing, sexual polymorphism and microsatellites in the hermaphroditic freshwater snail Bulinus truncatus. Proc. R. Soc. B, 264, $39-44$.

White, M. D. J. 1973. Animal Cytology and Evolution. Cambridge University Press, Cambridge.

woolhouse, M. E. J. AND CHANDiwANA, S. K. 1989. Spatial and temporal heterogeneity in the population dynamics of Bulinus globosus and Biomphalaria pfeifferi and the epidemiology of their infection with schistosomes. Parasitology, 98, $21-34$. 